Juria

\title{
Justizinitiative - Gegenvorschlag der Kommission für Rechtsfragen des Nationalrates
}

Der Bundesrat hat in seiner Botschaft zur «Justizinitiative» die Initiative ohne Gegenvorschlag zur Ablehnung empfohlen, obwohl er Verständnis für das Anliegen der Unterzeichnerinnen und Unterzeichner der Initiative äusserte. Die Kommission für Rechtsfragen des Nationalrates beschloss an ihrer Sitzung vom 6. November 2020 mit knapper Mehrheit einen indirekten Gegenentwurf zur Initiative.

Beitragsart: News $\mathrm{CH}$

Zitiervorschlag: Juria, Justizinitiative - Gegenvorschlag der Kommission für Rechtsfragen des Nationalrates, in: «Justice - Justiz - Giustizia» 2020/4 
[1] Am 8. September 2020 veröffentlichte der Bundesrat seine Botschaft zur am 19. August 2020 eingereichten Volksinitiative «Bestimmung der Bundesrichterinnen und Bundesrichter im Losverfahren (Justiz-Initiative)» ${ }^{1}$ sowie den Entwurf des Bundesbeschlusses über diese Volksinitiative $^{2}$. Nach Art. 1 Abs. 1 des E-Bundesbeschluss ist die Volksinitiative vom 26. August 2019 «Bestimmung der Bundesrichterinnen und Bundesrichter im Losverfahren (Justiz-Initiative)» gültig und wird Volk und Ständen zur Abstimmung unterbreitet.

[2] Gemäss Art. 1 Abs. 2 E-Bundesbeschluss lautet die Initiative wie folgt:

«Die Bundesverfassung wird wie folgt geändert:

Art. 145 Amtsdauer

${ }^{1}$ Die Mitglieder des Nationalrates und des Bundesrates sowie die Bundeskanzlerin oder der Bundeskanzler werden auf die Dauer von vier Jahren gewählt. Die Amtsdauer der Richterinnen und Richter des Bundesgerichts endet fünf Jahre nach Erreichen des ordentlichen Rentenalters.

2 Die Vereinigte Bundesversammlung kann auf Antrag des Bundesrates mit einer Mehrheit der Stimmenden eine Richterin oder einen Richter des Bundesgerichts abberufen, wenn diese oder dieser:

a. Amtspflichten schwer verletzt hat; oder

b. die Fähigkeit, das Amt auszuüben, auf Dauer verloren hat.

Art. 168 Abs. 1

${ }^{1}$ Die Bundesversammlung wählt die Mitglieder des Bundesrates, die Bundeskanzlerin oder den Bundeskanzler sowie den General.

Art. 188a Bestimmung der Richterinnen und Richter des Bundesgerichts

${ }^{1}$ Die Richterinnen und Richter des Bundesgerichts werden im Losverfahren bestimmt. Das Losverfahren ist so auszugestalten, dass die Amtssprachen im Bundesgericht angemessen vertreten sind.

${ }^{2}$ Die Zulassung zum Losverfahren richtet sich ausschliesslich nach objektiven Kriterien der fachlichen und persönlichen Eignung für das Amt als Richterin oder Richter des Bundesgerichts.

${ }^{3}$ Über die Zulassung zum Losverfahren entscheidet eine Fachkommission. Die Mitglieder der Fachkommission werden vom Bundesrat für eine einmalige Amtsdauer von zwölf Jahren gewählt. Sie sind in ihrer Tätigkeit von Behörden und politischen Organisationen unabhängig.

\footnotetext{
BB1 20206821.
}

2 BBl 20206847. 
Art. 197 Ziff. 12

12. Übergangsbestimmung zu den Art. 145 (Amtsdauer), 168 Abs. 1 und 188a (Bestimmung der Richterinnen und Richter des Bundesgerichts)

Ordentliche Richterinnen und Richter des Bundesgerichts, die bei Inkrafttreten der Artikel 145, 168 Absatz 1 und 188a im Amt sind, können noch bis zum Ende des Jahres, in dem sie das 68. Altersjahr vollenden, im Amt bleiben.»

[3] Am 6. November 2020 veröffentlichte die Kommission für Rechtsfragen des Nationalrates die folgende Medienmitteilung ${ }^{3}$ :

«Die RK-N hat die Volksinitiative 20.061 «Bestimmung der Bundesrichterinnen und Bundesrichter im Losverfahren (Justiz-Initiative). Volksinitiative` vorgeprüft, deren Ziel es ist, die Unabhängigkeit der Bundesrichterinnen und Bundesrichter gegenüber den politischen Parteien zu stärken, und zwar sowohl bei der Nominierung als auch bei der Wahl und der Wiederwahl. Um dieses Ziel zu erreichen, sieht die Initiative vor, dass die Richterinnen und Richter des Bundesgerichts mittels Losentscheid bestimmt werden, nachdem sie nach objektiven Kriterien von einer unabhängigen Fachkommission vorselektioniert wurden. Sie sollen sich nicht mehr zur Wiederwahl stellen müssen, sondern abberufen werden können, wenn sie ihre Amtspflichten schwer verletzt oder die Fähigkeit, das Amt auszuüben, verloren haben. Der Bundesrat beantragt dem Parlament in seiner Botschaft vom 19. August 2020, die Initiative zur Ablehnung zu empfehlen, obwohl er Verständnis für die Anliegen der Unterzeichnerinnen und Unterzeichner der Initiative hat.

Nachdem die Kommission gestern das Initiativkomitee und verschiedene Fachpersonen angehört hatte, führte sie heute Morgen eine eingehende Diskussion in dieser Sache. Wie der Bundesrat ist sie der Auffassung, dass die Unabhängigkeit der Richterinnen und Richter wichtig ist, die Initiative aber - insbesondere mit der Einführung des Losverfahrens - mehr Probleme schafft als löst: Das aktuelle System, d. h. die Wahl durch die Volksvertreterinnen und -vertreter, stellt sicher, dass die Richterinnen und Richter über die nötige Legitimität verfügen und ihre Entscheide akzeptiert werden. Zudem bietet es den Vorteil, eine ausgewogene Zusammensetzung des Bundesgerichts hinsichtlich Geschlecht, regionaler Herkunft und politischer Grundhaltung zu gewährleisten. Die Kommission ist überzeugt, dass die Unabhängigkeit der Justiz in der Schweiz sichergestellt ist und dass die Gerichtskommission, die mit der Vorbereitung der Richterwahlen betraut ist, ihre Aufgabe korrekt erfüllt. Sie räumt allerdings ein, dass Verbesserungen immer möglich sind und die Mandatsabgaben, die alle Richterinnen und Richter an ihre Partei entrichten, den Anschein erwecken können, diese seien von der Politik abhängig. Mit sehr knapper Mehrheit (13 zu 12 Stimmen) hat sie deshalb einen Antrag auf einen indirekten Gegenentwurf angenommen, der eine objektivere Auswahl der Richterinnen und Richter (Vorselektion durch eine Fachkommission einzig auf der Grundlage der fachlichen und persönlichen Eig-

3 https://www.parlament.ch/press-releases/Pages/mm-rk-n-2-2020-11-06.aspx. 
Juria, Justizinitiative - Gegenvorschlag der Kommission für Rechtsfragen des Nationalrates, in: «Justice - Justiz Giustizia» 2020/4

nung) und eine Prüfung von Alternativen zu den Mandatsabgaben vorsieht sowie die systematische Wiederwahl abschafft und die Abberufung der Bundesrichterinnen und Bundesrichter ermöglicht (20.480).»

[4] Der Gegenvorschlag der RK-N vom 6. November 2020 hat folgenden Wortlaut ${ }^{4}$ :

«Die Rechtsordnung soll dahingehend angepasst werden, dass die Richter*innen sämtlicher Gerichte des Bundes künftig wie folgt ausgewählt und gewählt werden:

- Die Wahl der Richter*innen erfolgt weiterhin durch die vereinigte Bundesversammlung auf Antrag der Gerichtskommission. Der Antrag der Gerichtskommission basiert neu mit / auf der Vorselektion durch eine Fachkommission. / Die Fachkommission berücksichtigt einzig die fachliche (inkl. Sprache) und persönliche Eignung der Kandidatinnen und Kandidaten. (evtl. 2-stufiges Verfahren)

- Die Wahl erfolgt für eine feste Amtsdauer von sechs Jahren. Die Wiederwahl erfolgt automatisch oder durch die Gerichtskommission auf Empfehlung der Fachkommission.

- Die Abberufung ist bei schwerer vorsätzlicher Pflichtverletzung und/oder Amtsunfähigkeit auf Antrag der Gerichtskommission jederzeit möglich. Die Fachkommission ist für die Erstellung des Sachverhalts zuständig.

- Die Unabhängigkeit der Richter*innen von den Parteien ist zu gewährleisten. Es sind Alternativen zu Mandatsabgaben zu prüfen.»

[5] Gemäss ihrer Medienmitteilung vom 6. November 2020 wird die RK-N ihre Arbeiten fortführen, sobald ihre ständerätliche Schwesterkommission dem Antrag auf einen indirekten Gegenentwurf zugestimmt hat.

4 Parlamentarische Initiative, Unabhängige und kompetente Richterinnen und Richter des Bundes. Indirekter Gegenvorschlag zur Justizinitiative, 20.480. 